\title{
Old proteins harbour unexpected functions
}

\author{
Rosario Perona
}

A $\mathrm{n}$ exciting fact in biology is the discovery of new and unsuspected roles for proteins whose function had been traditionally known for many years. This has been, in the past, the case for $\beta$-catenin, which was known originally as a cytoskeletal protein, before its role as a transcriptional regulator was found. It happened also with dyskerin, a pseudouridine synthase that modifies rRNA, which recently has been identified also as a component of an active telomerase complex. A recent finding identifies hTERT (a reverse transcriptase subunit of telomerase) as a modulator of Wnt signalling by association with target gene chromatin [1] and histone H2AX as an activator of hypoxia-dependent angiogenesis [2].

hTERT, together with its RNA subunit, hTR and dyskerin, constitutes a telomerase complex and synthesises telomere repeats. It is well known that telomerase homeostasis is important in cancer, since all tumours require increased levels of telomerase to counteract the telomere shortening that takes place with each cell division. This process is especially relevant in the preservation of mammalian tissues that require strict regulation of cell proliferation and differentiation of cells and activation of adult stem cells to facilitate organ homeostasis and guarantee repair of damaged organs. This is achieved by regulating the interaction of proteins of different signalling pathways in specific cell types. One of these pathways is controlled by Wnt proteins, which are crucial for development, stemcell maintenance and stem-cell activation. Park et al.'s

\author{
R. Perona $(\varangle)$ \\ Instituto de Investigaciones Biomédicas CSIC/UAM \\ Translational Oncology Unit \\ UAM/Hospital La Paz \\ C/ Arturo Duperier, 4 \\ ES-28029 Madrid, Spain \\ e-mail: rperona@iib.uam.es \\ R. Perona \\ CIBER de Enfermedades Raras \\ Valencia, Spain
}

study reports that telomerase functions in tissue progenitor cells to increase the transcription of genes activated by the Wnt- $\beta$-catenin signalling pathway [1]. Wnt proteins bind to membrane-bound Frizzled receptors and LRP co-receptors, preventing degradation of cytoplasmic $\beta$-catenin, which translocates to the nucleus, where it activates target genes by binding to LEF/TCF transcription factors. The first evidence linking hTERT to $\beta$-catenin was obtained in mice that expressed extra copies of the gene encoding TERT, which could be switched on in the adult stage of the animal in epithelial cells [3]. This study revealed that expression of TERT in these animals mimics the results obtained if active $\beta$-catenin was expressed in the mouse skin: an increase in proliferation in bulge stem cells and initiation of a new phase of hair growth [4]. Interestingly, this activity on hairfollicle growth was independent of the reverse transcriptase activity of hTERT, suggesting a non-canonical function for this protein. The explanation for the atypical function of hTERT was provided by Park et al. BRG1 is a subunit of a complex that facilitates transcription by altering chromatin conformation. B-catenin binds directly to BRG1 and TCF and increases transcription of $\beta$-catenin target genes. Unexpectedly, TERT was found forming complexes with BRG1, $\beta$-catenin and TCF in cells from mouse small intestine. Telomerase activity on telomere elongation is very relevant in stem cells to maintain the proliferative capacity and prevent senescence. By interacting with the $\beta$-catenin signalling pathway TERT can coordinate both processes: telomere integrity and cell proliferation, to regulate progenitor cell proliferation and renewal of tissues.

Another unexpected finding pertains to the histone family member H2AX. Upon DNA damage H2AX is phosphorylated in a conserved serine-glutamine motif and can mediate DNA repair by recruiting repairing and signalling factors to the DNA damaged site. The protein kinases ATR and ATM transduce the DNA damage signal to downstream effectors that alter cell cycle and cell survival responses depending on the magnitude of the damage. A recent study in Nature Medicine [2] provides evidence for a new role of $\mathrm{H} 2 \mathrm{AX}$ in tumorigenesis. These authors demonstrate that by genetic inactivation of $\mathrm{H} 2 \mathrm{AX}$, suppression of tumour angiogenesis is obtained in xenograft models. Additionally, inactivation of $\mathrm{H} 2 \mathrm{AX}$ in endothelial cells also suppresses tumour angiogenesis and growth, suggesting that the DNA damage response in endothelial cells is involved in tumour angiogenesis. Hypoxia, as DNA damage, is able to activate 
$\mathrm{H} 2 \mathrm{AX}$, therefore the authors examined if hypoxia contributes to the pro-angiogenic effect of H2AX. This study reveals that hypoxia is sufficient to induce H2AX phosphorylation, proliferation of endothelial cells and neovascularisation that includes pathological proliferative retinopathy and hind limb ischaemia. These findings demonstrate that $\mathrm{H} 2 \mathrm{AX}$ is crucial in hypoxia-induced angiogenesis and suggest that in addition to the transcriptional induction of pro-angiogenic gene expression hypoxia can also influence angiogenesis through alternative routes such as the DNA repair pathway. Elucidating the intimate mechanism of $\mathrm{H} 2 \mathrm{AX}$-induced angiogenesis will influence and help development of new angiogenic inhibitors.

All these findings demonstrate that basic research often takes unexpected turns that bring about changes in our understanding of key biological processes such as cancer onset and progression. The lesson is quite remarkable and deserves consideration. Even if we feel that we have exhausted the repertoire of molecular targets, this kind of finding demonstrates that we still have many bullets to shoot in our battle against cancer.

\section{References}

1. Park JI, Venteicher AS, Hong JY et al (2009) Telomerase modulates Wnt signalling by association with target gene chromatin. Nature 4460:66-72

2. Economopoulou M, Langer HF, Celeste A et al (2009) Histone H2AX is integral to hypoxia-driven neovascularization. Nat Med 14:553-558

3. Sarin KY, Cheung P, Gilison D et al (2005) Conditional telomerase induction causes proliferation of hair follicle stem cells. Nature 436:1048-1052

4. Choi J, Southworth LK, Sarin KY et al (2008) TERT promotes epithelial proliferation through transcriptional control of a Myc- and Wnt-related developmental program. PLOS Genet 4:0124-0138 